\title{
Efetividade de herbicidas no controle de Alternanthera tenella
}

\author{
Effectiveness of herbicides for Alternanthera tenella control
}

\author{
Rosecler Salete Canossa ${ }^{1}$, Rubem Silvério de Oliveira Jr. ${ }^{2}$, Jamil Constantin ${ }^{2}$, \\ Fabiano Aparecido Rios ${ }^{3}$, Sidnei Douglas Cavalieri ${ }^{4}$
}

\begin{abstract}
Resumo - O objetivo deste trabalho foi avaliar a eficácia de diferentes herbicidas, aplicados em pré e pós-emergência, no controle de Alternanthera tenella. Foram realizados dois ensaios em casa-de-vegetação: no primeiro, foram avaliados herbicidas aplicados em pré-emergência, e no segundo foram avaliados herbicidas em pós-emergência, aplicados quando a planta daninha apresentava dois pares de folhas. Os tratamentos em pré-emergência e as respectivas doses ( $\mathrm{g} \mathrm{ha}^{-}$ ${ }^{1}$ ) avaliadas foram: alachlor (2880); chlorimuron-ethyl (15); metribuzin (576); clomazone (1000); flumetsulam (120); sulfentrazone (600); trifluralin (540); amicarbazone (1050); pendimethalin (1500); oxyfluorfen (360); ametryn (1500); diuron (1500); imazapic (140); isouron (175); smetolachlor (576 e 960) e testemunha sem aplicação. Os tratamentos avaliados em pósemergência foram: fomesafen (250); [diuron+paraquat] [200+400]; 2,4-D (1005); carfentrazoneethyl (60); chlorimuron-ethyl (15); lactofen (168); bentazon (720); flumiclorac-pentyl (60); [glyphosate+imazethapyr] [444,5+75]; diquat (400); glyphosate (540 e 1440) e testemunha sem aplicação. Nas aplicações em pré-emergência, todos os herbicidas foram considerados eficazes para o controle de A. tenella, proporcionando controle acima de $98 \%$ aos 28 dias após aplicação (DAA). Os resultados das aplicações pós-emergentes demonstraram que os herbicidas fomesafen, lactofen e bentazon não foram eficazes no controle de A. tenella. Os demais herbicidas aplicados em pós-emergência foram altamente eficazes, resultando num controle acima de $97 \%$ aos 28 DAA.
\end{abstract}

Palavras-chave: Apaga-fogo, controle químico, pré-emergência, pós-emergência.

\begin{abstract}
The objective of this work was to evaluate the efficacy of different herbicides, applied both at pre and post-emergence, to control Alternanthera tenella. Two assays were accomplished: the first one for pre-emergence herbicides, and the second one for post-emergence herbicides, applied at two pair of leaves stage. Pre-emergence herbicide treatments and respective rates (g i.a. $\mathrm{ha}^{-1}$ ) were: alachlor (2880); chlorimuron-ethyl (15); metribuzin (576); clomazone (1000); flumetsulam (120); sulfentrazone (600); trifluralin (540); amicarbazone (1050); pendimethalin (1500); oxyfluorfen (360); ametryn (1500); diuron (1500); imazapic (140); isouron

\footnotetext{
${ }^{1}$ Discente do Programa de Pós-Graduação em Agronomia da Universidade Estadual de Maringá (UEM/NAPD), Av. Colombo 5790, 87020-900, Maringá, PR.

${ }^{2}$ Professor Associado, Departamento de Agronomia da UEM. Av. Colombo 5790 87020-900 Maringá, PR. E-mail: rsojunior@uem.br

${ }^{3}$ Professor Discente do Programa de Pós-Graduação em Agronomia da Universidade Estadual de Maringá (UEM/NAPD), Av. Colombo 5790 87020-900 Maringá, PR.

${ }^{4}$ Pesq. Dr. Embrapa Hortaliças/CNPH, Rodovia Brasilia/Anapolis, BR 060 Km 09 Gama/DF, CP 218, CEP 70359-970.
}

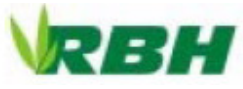


(175); s-metolachlor (576 and 960) and check plot. Post-emergence herbicide treatments were: fomesafen (250); [diuron+paraquat] [200+400]; 2,4-D (1005); carfentrazone-ethyl (60); chlorimuron-ethyl (15); lactofen (168); bentazon (720); flumiclorac-pentyl (60); [glyphosate+imazethapyr] [444,5+75]; diquat (400); glyphosate (540 and 1440) and check plot. For pre-emergence applications, all herbicides were considered efficient for A. tenella control, propitiating more than $98 \%$ control at 28 days after application (DAA). The post-emergence herbicide treatments, fomesafen, lactofen and bentazon did not control A. tenella. Remaining post-emergence treatments provided more than $97 \%$ control of $A$. tenella at 28 DAA.

Key-words: Joyweed, Calico plant, chemical control, pre-emergence, post-emergence.

\section{Introdução}

Alternathera tenella, conhecida como apaga-fogo, é uma planta herbácea, muito ramificada, tendendo a formar uma cobertura intensa sobre o solo. Esta espécie alastra-se por enraizamento a partir de nós em contato com o solo. A. tenella ocorre como infestante de pastagens e de culturas anuais, sendo particularmente prejudicial como infestante tardia, que pode dificultar a colheita mecânica e aumentar o teor de impureza e de umidade dos grãos colhidos. Segundo Kissmann \& Groth (1999), A. tenella apresenta o ciclo $\mathrm{C}_{4}$ da fotossíntese, sendo muito competitiva, especialmente quando ocorre em lavouras de plantas que apresentam o ciclo $\mathrm{C}_{3}$.

Há relatos de infestações de $A$. tenella em diversas culturas e regiões do Brasil. Freitas et al. (2006), relatam a principal espécie de planta daninha encontrada na cultura de algodão em Minas Gerais foi $A$. tenella, e que esta apresentava a maior densidade de infestação $\left(127,4\right.$ plantas $\left.\mathrm{m}^{-2}\right)$ dentre as espécies presentes na área. Pereira \& Velini (2003) avaliaram a flora infestante no município de Chapadão do Céu, GO, durante quatro anos (1995 a 1998) e constataram a presença de A. tenella como uma das espécies de maior importância. Segundo Carvalho et al. (2005) em estudo de plantas infestantes na cultura de citros em pomares no Estado de
Sergipe, A. tenella se destacou entre as plantas daninhas que apresentaram maior frequência de ocorrência no período da pesquisa. Segundo Lacerda (2003), dentre as plantas daninhas estudadas em sistema de plantio direto e convencional no estado de São Paulo, a A. tenella foi considerada uma das espécies mais frequentes. Diversos outros trabalhos relatam A. tenella como infestante de grande relevância em áreas de milho, soja, algodão e tomate (Duarte \& Deuber, 1999; Nascente et al., 2004; Bertin et al., 2005; Timossi et al., 2006). De acordo com Shiratsuchi et al. (2001), a espécie é considerada como uma das plantas daninhas mais problemáticas na cultura da soja quando não controlada adequadamente. Estes autores avaliaram a emergência de plantas daninhas na cultura de soja no estado de São Paulo, e encontraram um banco de 178,4 sementes $\mathrm{m}^{-2}$, sendo ainda que o método utilizado para determinação do banco de sementes não contabilizou as sementes dormentes. Além de infestante de culturas, pesquisas relatam A. tenella como hospedeira de bactérias. Segundo Lopes et al. (2003) essa espécie foi detectada como hospedeira da bactéria Xylella fastidiosa, causadora da clorose variegada dos citrus (CVC).

Embora existam diversos herbicidas registrados para uso no controle desta espécie (Rodrigues \& Almeida, 2005), as informações 
sobre a eficácia destes herbicidas no controle de A. tenella são relativamente limitadas, e existem evidências de controle deficiente e de aumento das áreas infestadas com esta espécie nas áreas de plantio direto, principalmente em áreas de produção de grãos no Cerrado brasileiro.

O herbicida imazamox (30 $\left.\mathrm{g} \mathrm{ha}^{-1}\right)$ aplicado em pós-emergência (dois pares de folhas definitivas) promoveu controle de $87,50 \%$ de A. tenella (Correia et al., 2005). Controle eficiente também foi observado pela aplicação pré-emergente de flumioxazin $(50 \mathrm{~g}$ $\mathrm{ha}^{-1}$ ) (Jaremtchuck et al., 2006) e pela aplicação pós-emergente de trifloxysulfuronsodium, em doses de 8 e $10 \mathrm{~g} \mathrm{ha}^{-1}$ (Peixoto et al., 2006). Resultados semelhantes foram observados por Braz et al. (2002) ao utilizar a associação de trifloxysulfuron-sodium + ametrina $\left(37+1465 \mathrm{~g} \mathrm{ha}^{-1}\right)$, os quais apresentaram excelente controle (>90\%) 156 dias após aplicação sobre plantas no estádio de três folhas. Segundo as observações de Foloni \& Bachiega (2002), a associação do mesotrione + atrazine também foi eficaz para o controle de A. tenella quando aplicada no estádio de 1-4 folhas. A associação de herbicidas [bentazon + paraquat] com imazetapyr ou lactofen aumentou o índice de controle para todas as espécies de plantas daninhas estudadas por Rozanski et al. (2004), dentre elas, A. tenella. Por outro lado, Negrisoli et al. (2003) não obtiveram sucesso no controle desta espécie com a utilização de 2,4-D e glyphosate associados à atrazine, em infestação de 82,5 plantas $\mathrm{m}^{-2}$.

O objetivo deste trabalho foi avaliar a efetividade de diferentes herbicidas, aplicados em pré e pós-emergência, no controle de A. tenella.

\section{Material e métodos}

Dois experimentos foram desenvolvidos em casa de vegetação da Universidade Estadual de Maringá, no ano de 2006. O solo utilizado nos experimentos apresentava textura arenosa, com $920 \mathrm{~g} \mathrm{~kg}^{-1}$ de areia; $30 \mathrm{~g}$ $\mathrm{kg}^{-1}$ de argila; 7,36 $\mathrm{g} \mathrm{dm}^{-3}$ de $\mathrm{Ce} \mathrm{pH}\left(\mathrm{H}_{2} \mathrm{O}\right)=$ 6,2. A umidade do solo foi reposta diariamente. Foram colocadas 20 sementes por vaso (capacidade de $5 \mathrm{~kg}$ de solo) de $A$. tenella, na profundidade de $1 \mathrm{~cm}$.

No primeiro experimento, foram avaliados herbicidas aplicados em préemergência, e no segundo herbicidas aplicados em pós-emergência. Em ambos os experimentos a aplicação dos herbicidas foi realizada utilizando-se um pulverizador costal de pressão constante à base de $\mathrm{CO}_{2}$, equipado com pontas TJ 11002 - pressão de $30 \mathrm{lb} \mathrm{pol}^{-}$ ${ }^{2}$, sendo que a altura da aplicação foi $50 \mathrm{~cm}$ acima da borda dos vasos. O volume de calda aplicado foi equivalente a $200 \mathrm{~L} \mathrm{ha}^{-1}$. As condições ambientais no momento da aplicação para ambos experimentos foram de umidade relativa do ar de $78 \%$, temperatura ambiente de $29^{\circ} \mathrm{C}$ e velocidade do vento inferior a $4 \mathrm{~km} \mathrm{~h}^{-1}$.

\section{a) Controle de Alternanthera tenella em condições de pré-emegência}

Os tratamentos avaliados neste experimento foram constituídos de 17 tratamentos (Tabela 1). As doses utilizadas foram baseadas nas recomendações de Rodrigues e Almeida (2005). A aplicação dos herbicidas foi realizada no mesmo dia da semeadura. O controle de A. tenella foi avaliado por meio de escala percentual de notas aos 14, 21 e 28 dias após aplicação (DAA). O valor 0 (zero) correspondeu a nenhum controle e $100 \%$ à morte total das plantas, comparados à testemunha sem aplicação de herbicida. $\mathrm{O}$ delineamento experimental foi em blocos casualizados com três repetições, sendo cada unidade experimental constituída por um vaso. Os 
dados obtidos foram submetidos à análise de variância e as médias comparadas pelo teste de Tukey a 5\% de probabilidade.

\section{b) Controle de Alternanthera tenella em condições de pós-emergência}

A aplicação foi realizada quando as plantas de apaga-fogo apresentavam $\mathrm{o}$ segundo par de folhas verdadeiras completamente expandidas, que ocorreu aos 21 dias após semeadura, quando as plantas apresentavam altura média de $3,5 \mathrm{~cm}$. Os herbicidas e as respectivas doses avaliadas neste experimento estão descritos na Tabela 2.

As doses utilizadas foram baseadas nas recomendações de Rodrigues e Almeida (2005).

Tabela 1. Herbicidas utilizados para aplicação em pré-emergência visando o controle de Alternanthera tenella.

\begin{tabular}{|c|c|c|c|c|}
\hline Tratamentos & $\begin{array}{l}\text { Dose } \\
\left(\mathrm{g} \mathrm{ha}^{-1}\right)\end{array}$ & Produto comercial & $\begin{array}{c}\text { Concentração } \\
\left(\mathrm{g} \mathrm{L}^{-1} \mathrm{ou} \mathrm{g} \mathrm{kg}^{-1}\right)\end{array}$ & $\begin{array}{c}\text { Dose } \\
\left(\mathrm{g} \mathrm{ou} \mathrm{L} \mathrm{ha}^{-1}\right)\end{array}$ \\
\hline alachlor & 2880 & Alaclor Nortox ${ }^{\circledR}$ & $480 \mathrm{~g} \mathrm{~L}^{-1}$ & $6,00 \mathrm{~L}$ \\
\hline chlorimuron-ethyl & 15 & Classic $^{\circledR}$ & $250 \mathrm{~g} \mathrm{~kg}^{-1}$ & $60,00 \mathrm{~g}$ \\
\hline metribuzin & 576 & Sencor $480^{\circledR}$ & $480 \mathrm{~g} \mathrm{~L}^{-1}$ & $1,20 \mathrm{~L}$ \\
\hline clomazone & 1000 & Gamit $^{\circledR}$ & $500 \mathrm{~g} \mathrm{~L}^{-1}$ & $2,00 \mathrm{~L}$ \\
\hline flumetsulam & 120 & Scorpion $^{\circledR}$ & $120 \mathrm{~g} \mathrm{~L}^{-1}$ & $1,00 \mathrm{~L}$ \\
\hline sulfentrazone & 600 & Boral $500 \mathrm{SC}^{\circledR}$ & $500 \mathrm{~g} \mathrm{~L}^{-1}$ & $1,20 \mathrm{~L}$ \\
\hline trifluralin & 540 & Trifluralina Nortox Gold ${ }^{(B)}$ & $450 \mathrm{~g} \mathrm{~L}^{-1}$ & $1,20 \mathrm{~L}$ \\
\hline amicarbazone & 1050 & Dinamic $^{\circledR}$ & $700 \mathrm{~g} \mathrm{~kg}^{-1}$ & $1,50 \mathrm{~kg}$ \\
\hline pendimethalin & 1500 & Herbadox $500 \mathrm{CE}^{\circledR}$ & $500 \mathrm{~g} \mathrm{~L}^{-1}$ & $3,00 \mathrm{~L}$ \\
\hline oxyfluorfen & 360 & Goal BR ${ }^{\circledR}$ & $240 \mathrm{~g} \mathrm{~L}^{-1}$ & $1,50 \mathrm{~L}$ \\
\hline ametryn & 1500 & Herbipak $500 \mathrm{BR}^{\circledR}$ & $500 \mathrm{~g} \mathrm{~L}^{-1}$ & $3,00 \mathrm{~L}$ \\
\hline diuron & 1500 & Herburon $500 \mathrm{BR}^{\circledR}$ & $500 \mathrm{~g} \mathrm{~L}^{-1}$ & $3,00 \mathrm{~L}$ \\
\hline imazapic & 140 & Plateau $^{\circledR}$ & $700 \mathrm{~g} \mathrm{~kg}^{-1}$ & $0,20 \mathrm{~kg}$ \\
\hline isouron & 175 & * & $500 \mathrm{~g} \mathrm{~kg}^{-1}$ & $0,35 \mathrm{~kg}$ \\
\hline s-metolachlor & 576 & Dual Gold $^{\circledR}$ & $960 \mathrm{~g} \mathrm{~L}^{-1}$ & $0,60 \mathrm{~L}$ \\
\hline s-metolachlor & 960 & Dual Gold $^{\circledR}$ & $960 \mathrm{~g} \mathrm{~L}^{-1}$ & $1,00 \mathrm{~L}$ \\
\hline $\begin{array}{l}\text { Testemunha sem } \\
\text { aplicação }\end{array}$ & - & - & - & - \\
\hline
\end{tabular}

* Herbicida em fase de avaliação.

O controle na modalidade pós- obtidos foram submetidos à análise de variância emergência foi avaliado por meio de escala e as médias comparadas pelo teste de Tukey a percentual de notas aos 3, 7, 14, 21 e 28 dias $5 \%$ de probabilidade.

após aplicação (DAA), onde $0 \%$ (zero) corresponde a nenhum controle e $100 \%$ à morte das plantas, comparados à testemunha sem aplicação de herbicida. O delineamento experimental foi em blocos casualizados com quatro repetições, sendo cada unidade

\section{Resultados e discussão}

a) Controle de Alternanthera tenella em préemegência experimental constituída por um vaso. Os dados 
Os resultados de porcentagem de controle aos 14, 21 e 28 dias após aplicação (DAA) estão descritos na Tabela 3. Todos os herbicidas avaliados foram eficazes para o controle de Alternanthera tenella, sendo que a maioria dos herbicidas proporcionou $100 \%$ de controle aos 28 DAA. Os únicos herbicidas que não apresentaram controle total foram diuron (98,5\%), flumetsulam e ametryn (99\%), imazapic e isouron $(99,50 \%)$, e s-metolachlor nas duas doses $(99,75 \%)$, sem que, no entanto, houvesse diferenças significativas nos níveis de controle.

Tabela 2. Herbicidas utilizados para aplicação em pós-emergência visando controle de Alternanthera tenella.

\begin{tabular}{|c|c|c|c|c|}
\hline Tratamentos & $\begin{array}{l}\text { Dose } \\
\left(\mathrm{g} \mathrm{ha}^{-1}\right)\end{array}$ & Produto comercial & 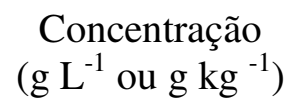 & $\begin{array}{c}\text { Dose } \\
\left(\mathrm{g} \mathrm{ou} \mathrm{L} \mathrm{ha}^{-1}\right)\end{array}$ \\
\hline fomesafen $^{\frac{1}{}}$ & 250 & Flex $^{\circledR}$ & $250 \mathrm{~g} \mathrm{~L}^{-1}$ & $1,00 \mathrm{~L}$ \\
\hline [diuron + paraquat] & {$[200+400]$} & Gramocil $^{\circledR}$ & $100+200 \mathrm{~g} \mathrm{~L}^{-1}$ & $2,00 \mathrm{~L}$ \\
\hline $2,4-\mathrm{D}$ & 1005 & DMA 806 BR $^{\circledR}$ & $670 \mathrm{~g} \mathrm{~L}^{-1}$ & $1,50 \mathrm{~L}$ \\
\hline carfentrazone-ethyl & 60 & Aurora $^{\circledR}$ & $400 \mathrm{~g} \mathrm{~L}^{-1}$ & $0,15 \mathrm{~L}$ \\
\hline chlorimuron-ethyl ${ }^{2 /}$ & 15 & Classic $^{\circledR}$ & $250 \mathrm{~g} \mathrm{~kg}^{-1}$ & $60,00 \mathrm{~g}$ \\
\hline lactofen & 168 & $\mathrm{Naja}^{\circledR}$ & $240 \mathrm{~g} \mathrm{~L}^{-1}$ & $0,70 \mathrm{~L}$ \\
\hline bentazon ${ }^{3 /}$ & 720 & Basagran $600^{\circledR}$ & $600 \mathrm{~g} \mathrm{~L}^{-1}$ & $1,20 \mathrm{~L}$ \\
\hline flumiclorac-pentyl ${ }^{4 /}$ & 60 & Radiant $100^{\circledR}$ & $100 \mathrm{~g} \mathrm{~L}^{-1}$ & $0,60 \mathrm{~L}$ \\
\hline $\begin{array}{l}\text { [glyphosate + } \\
\text { imazethapyr] }\end{array}$ & {$[444,5+75]$} & Alteza $30 \mathrm{SL}^{\circledR}$ & $177,8+30 \mathrm{~g} \mathrm{~L}^{-1}$ & $2,50 \mathrm{~L}$ \\
\hline diquat & 400 & $\begin{array}{l}\text { Reglone }^{\circledR} \\
\text { Round up }\end{array}$ & $200 \mathrm{~g} \mathrm{~L}^{-1}$ & $2,00 \mathrm{~L}$ \\
\hline glyphosate & 540 & $\begin{array}{l}\text { Original }^{\circledR} \\
\text { Round up }\end{array}$ & $360 \mathrm{~g} \mathrm{~L}^{-1}$ & $1,50 \mathrm{~L}$ \\
\hline glyphosate & 1440 & Original $^{\circledR}$ & $360 \mathrm{~g} \mathrm{~L}^{-1}$ & $4,00 \mathrm{~L}$ \\
\hline $\begin{array}{l}\text { Testemunha sem } \\
\text { aplicação }\end{array}$ & - & - & - & - \\
\hline $\begin{array}{l}{ }^{1 /} \text { Acrescentou-se En } \\
{ }^{2 /} \text { Acrescentou-se As } \\
\text { 3/ Acrescentou-se Da } \\
{ }^{4 /} \text { Acrescentou-se Dy }\end{array}$ & $\begin{array}{l}\text { a } 0,5 \% \mathrm{v} / \mathrm{v} . \\
0,05 \% \mathrm{v} / \mathrm{v} . \\
, 5 \% \mathrm{v} / \mathrm{v} . \\
0,25 \% \mathrm{v} / \mathrm{v} .\end{array}$ & & & \\
\hline
\end{tabular}

Embora controle total aos 28 DAA tenha sido observado pela aplicação em préemergência do herbicida chlorimuron-ethyl, este produto não é recomendado nesta modalidade de aplicação (Lorenzi, 2006). Também segundo de acordo com este autor, A. tenella é pouco suscetível (menos que 50\% de controle) ao herbicida clomazone, sendo que, neste trabalho foi observado controle total das plantas (Tabela 3). É possível que a textura arenosa do solo deste experimento tenha propiciado uma maior efetividade do chlorimuron e do clomazone no controle do apaga-fogo. $\mathrm{O}$ aumento do controle do apaga- 
fogo em solos de textura mais leve foi igualmente descrito pela utilização do flumioxazin (Jaremtchuck et al., 2006).

$\mathrm{Na}$ dose de $600 \mathrm{~g} \mathrm{ha}^{-1}$ o herbicida sulfentrazone controlou a espécie A. tenella com $100 \%$ de eficácia aos 28 DAA, como pode ser observado na Tabela 3. Dados disponibilizados por Christoffoleti et al. (2002) sugerem que doses ainda mais baixas deste herbicida $\left(150\right.$ a $\left.450 \quad \mathrm{~g} \mathrm{ha}^{-1}\right)$, também

Tabela 3. Porcentagens de controle de Alternanthera tenella observadas em três avaliações após a aplicação de herbicidas em pré-emergência.

\begin{tabular}{lcrrrr}
\hline & Dose & \multicolumn{5}{c}{ Controle $(\%)$} \\
\cline { 3 - 6 } \multicolumn{1}{c}{ Tratamentos } & $\left(\mathrm{g} \mathrm{ha}^{-1}\right)$ & $14 \mathrm{DAA}$ & $21 \mathrm{DAA}$ & $28 \mathrm{DAA}$ \\
\hline alachlor & 2880 & $100,00 \mathrm{a}$ & $100,00 \mathrm{a}$ & $100,00 \mathrm{a}$ \\
chlorimuron-ethyl & 15 & $99,75 \mathrm{a}$ & $99,00 \mathrm{ab}$ & $100,00 \mathrm{a}$ \\
metribuzin & 576 & $98,50 \mathrm{ab}$ & $100,00 \mathrm{a}$ & $100,00 \mathrm{a}$ \\
clomazone & 1000 & $99,50 \mathrm{a}$ & $99,50 \mathrm{a}$ & $100,00 \mathrm{a}$ \\
flumetsulam & 120 & $96,75 \mathrm{~b}$ & $97,75 \mathrm{~b}$ & $99,00 \mathrm{a}$ \\
sulfentrazone & 600 & $100,00 \mathrm{a}$ & $100,00 \mathrm{a}$ & $100,00 \mathrm{a}$ \\
trifluralin & 540 & $99,75 \mathrm{a}$ & $100,00 \mathrm{a}$ & $100,00 \mathrm{a}$ \\
amicarbazone & 1050 & $100,00 \mathrm{a}$ & $99,50 \mathrm{a}$ & $100,00 \mathrm{a}$ \\
pendimethalin & 1500 & $100,00 \mathrm{a}$ & $99,25 \mathrm{ab}$ & $100,00 \mathrm{a}$ \\
oxyfluorfen & 360 & $100,00 \mathrm{a}$ & $100,00 \mathrm{a}$ & $100,00 \mathrm{a}$ \\
ametryn & 1500 & $100,00 \mathrm{a}$ & $100,00 \mathrm{a}$ & $99,00 \mathrm{a}$ \\
diuron & 1500 & $99,50 \mathrm{a}$ & $99,00 \mathrm{ab}$ & $98,50 \mathrm{a}$ \\
imazapic & 140 & $100,00 \mathrm{a}$ & $99,50 \mathrm{a}$ & $99,50 \mathrm{a}$ \\
isouron & 145 & $100,00 \mathrm{a}$ & $99,50 \mathrm{a}$ & $99,50 \mathrm{a}$ \\
s-metolachlor & 576 & $100,00 \mathrm{a}$ & $100,00 \mathrm{a}$ & $99,75 \mathrm{a}$ \\
s-metolachlor & 960 & $100,00 \mathrm{a}$ & $100,00 \mathrm{a}$ & $99,75 \mathrm{a}$ \\
Testemunha sem aplicação & - & $0,00 \mathrm{c}$ & $0,00 \mathrm{c}$ & $0,00 \mathrm{~b}$ \\
\hline F & - & $4450,15 *$ & 6041,84 & 6387,26 & $*$ \\
CV (\%) & - & 0,77 & & 0,66 & 0,64 \\
DMS & - & 1,87 & 1,60 & 1,56
\end{tabular}

* As médias seguidas pelas mesmas letras na coluna não diferem entre si pelo teste de Tukey a $5 \%$ de probabilidade.

Também o s-metolachor $(576$ e $960 \mathrm{~g}$ $\mathrm{ha}^{-1}$ ) foi eficaz, apresentando $99,75 \%$ de controle. O mesmo foi observado por Freitas et al. (2006) para o controle de A. tenella com o herbicida s-metolachor na dose $1152 \mathrm{~g} \mathrm{ha}^{-1}$ observando-se controle superior a $90 \%$. O oxyfluorfen a $600 \mathrm{~g} \mathrm{ha}^{-1}$ proporcionou controle de $100 \%$ nesse experimento. Bezutte et al. propiciam controle adequado desta espécie. Trifluralin aplicado a $540 \mathrm{~g} \mathrm{ha}^{-1}$ promoveu controle total das plantas aos 28 DAA. Este resultado corrobora aqueles apresentados por Shiratsuchi (2001), que também relata que $A$. tenella foi susceptível à aplicação préemergente do trifluralin. 
controle observado mesmo em solo de textura leve.

\section{b) Controle de Alternanthera tenella em condições de pós-emergência}

Na primeira avaliação, realizada aos três dias após aplicação (DAA), observou-se controle superior a $80 \%$ de controle para os herbicidas [diuron + paraquat], carfentrazoneethyl, flumiclorac-penthyl e diquat, que apresentam ação predominantemente de contato (Tabela 4). No entanto, três herbicidas que também apresentam ação de contato não proporcionaram níveis aceitáveis de controle nesta avaliação (fomesafen, lactofen e bentazon). A baixa efetividade inicial destes três herbicidas foi ratificada nas avaliações aos 7 e aos 28 DAA. Valério et al. (2002) observaram que o lactofen nas doses 86, $120 \mathrm{e}$ $168 \mathrm{~g} \mathrm{ha}^{-1}$, foi eficaz para o controle de apagafogo, entretanto, a mistura do lactofen + imazetapyr ou chlorimuron, apresentaram índices superiores ao oferecido pelo lactofen aplicado isoladamente. Para aplicações em pós-emergência, Lorenzi (2006) considera $A$. tenella pouco suscetível ao herbicida bentazon, altamente suscetível ao fomesafen e suscetível ao lactofen.

Segundo Ferreira et al. (2003), A. tenella apresenta potenciais barreiras foliares que podem comprometer a absorção foliar dos herbicidas e, consequentemente, a eficácia de herbicidas aplicados em pós-emergência. Estes autores sugerem que a grande espessura da cutícula das faces abaxial e adaxial, o elevado teor de cera especuticular e a alta densidade de tricomas seriam os principais obstáculos para a absorção foliar dos herbicidas. Sugerem ainda que, para a espécie estudada, a adição de adjuvantes que possibilitem maior grau de contato de calda com a superfície foliar e o aumento de penetração estomática poderiam se constituir em estratégias importantes para maximizar a absorção foliar dos herbicidas. No entanto, no presente trabalho, tanto o fomesafen quanto $\mathrm{o}$ bentazon receberam adjuvantes para maximizar a absorção foliar e, mesmo assim, não conseguiram promover níveis de controle aceitáveis. Os demais herbicidas com ação de contato aplicados em pós-emergência, [diuron + paraquat], carfentrazone-ethyl, flumiclorac-penthyl e diquat, apresentaram controle praticamente total a partir de 7 DAA (Tabela 4). Segundo Lorenzi (2006), apenas o carfentrazone-ethyl está sem informação sobre a aplicação para $A$. tenella. Para os demais herbicidas estudados, os controles obtidos apresentaram-se de acordo com a classificação de susceptibilidade oferecida.

Os herbicidas de ação sistêmica como 2,4-D, chlorimuron-ethyl e [glyphosate + imazetapyr], em geral, apresentaram baixos valores de controle nas avaliações iniciais (3 e 7 DAA), mas aos 28 DAA a eficácia de controle destes tratamentos se assemelhava aos melhores tratamentos com herbicidas de ação de contato (Tabela 4). Estes resultados corroboram as informações de Lorenzi (2006) para os herbicidas 2,4-D e chlorimuron-ethyl. Em relação ao glyphosate aplicado isoladamente, observou-se acima de 90\% de eficácia para as duas doses avaliadas já aos 7 DAA. A partir dos 14 DAA, estes tratamentos assim como a mistura formulada [glyphosate + imazetapyr], proporcionaram controle total de A. tenella (Tabela 4). Resultado semelhante foi observado por Kawaguchi et al. (2003), onde o glyphosate aplicado na mesma dose e também em doses menores, proporcionou excelente controle para A. tenella. 
Tabela 4. Porcentagens de controle de Alternanthera tenella observadas em cinco avaliações após a aplicação de herbicidas em pósemergência.

\begin{tabular}{|c|c|c|c|c|c|c|}
\hline \multirow[b]{2}{*}{ Tratamento } & \multirow{2}{*}{$\begin{array}{c}\text { Dose } \\
\left(\mathrm{g} \mathrm{ha}^{-1}\right)\end{array}$} & \multicolumn{5}{|c|}{ Controle $(\%)$} \\
\hline & & 3 DAA & 7 DAA & 14 DAA & $21 \mathrm{DAA}$ & 28 DAA \\
\hline fomesafen $^{1 /}$ & 250 & $6,67 \mathrm{de}$ & $10,00 \mathrm{e}$ & $8,33 \mathrm{c}$ & $5,00 \mathrm{c}$ & $0,00 \mathrm{~d}$ \\
\hline [diuron + paraquat] & {$[200+400]$} & 94,33 a & $100,00 \mathrm{a}$ & $100,00 \mathrm{a}$ & $100,00 \mathrm{a}$ & $100,00 \mathrm{a}$ \\
\hline $2,4-d$ & 1005 & $35,00 \mathrm{bc}$ & $40,00 \mathrm{~d}$ & $63,33 \mathrm{~b}$ & $91,67 \mathrm{a}$ & 99,67 a \\
\hline carfentrazone-ethyl & 6 & 81,67 a & $60,00 \mathrm{~cd}$ & 98,33 a & $100,00 \mathrm{a}$ & $100,00 \mathrm{a}$ \\
\hline chlorimuron-ethyl ${ }^{\underline{2} /}$ & 15 & 6,67 de & $15,00 \mathrm{e}$ & $50,00 \mathrm{~b}$ & 90,00 a & 97,33 a \\
\hline lactofen & 168 & $45,00 \mathrm{~b}$ & $58,33 \mathrm{~cd}$ & $53,33 \mathrm{~b}$ & $58,33 \mathrm{~b}$ & $56,67 \mathrm{~b}$ \\
\hline bentazon ${ }^{3 /}$ & 720 & $36,67 \mathrm{bc}$ & $58,33 \mathrm{~cd}$ & $66,67 \mathrm{~b}$ & $53,33 \mathrm{~b}$ & $40,00 \mathrm{c}$ \\
\hline flumiclorac-pentyl ${ }^{4 /}$ & 60 & $91,00 \mathrm{a}$ & $100,00 \mathrm{a}$ & $99,67 \mathrm{a}$ & $100,00 \mathrm{a}$ & $100,00 \mathrm{a}$ \\
\hline [glyphosate + imazethapyr] & {$[444,5+75,0]$} & $36,67 \mathrm{bc}$ & $71,67 \mathrm{bc}$ & $100,00 \mathrm{a}$ & $100,00 \mathrm{a}$ & $100,00 \mathrm{a}$ \\
\hline diquat & 400 & $97,67 \mathrm{a}$ & $100,00 \mathrm{a}$ & $100,00 \mathrm{a}$ & $100,00 \mathrm{a}$ & $100,00 \mathrm{a}$ \\
\hline glyphosate & 540 & $23,33 \mathrm{~cd}$ & $91,67 \mathrm{ab}$ & $100,00 \mathrm{a}$ & $100,00 \mathrm{a}$ & $100,00 \mathrm{a}$ \\
\hline glyphosate & 1440 & $53,33 \mathrm{~b}$ & $95,00 \mathrm{a}$ & $100,00 \mathrm{a}$ & $100,00 \mathrm{a}$ & $100,00 \mathrm{a}$ \\
\hline Testemunha sem aplicação & - & $0,00 \mathrm{e}$ & $0,00 \mathrm{e}$ & $0,00 \mathrm{c}$ & $0,00 \quad \mathrm{c}$ & $0,00 \mathrm{~d}$ \\
\hline $\mathrm{F}$ & - & $95,25^{*}$ & $81,09 *$ & $37,38^{*}$ & $166,80 *$ & $304,77 *$ \\
\hline $\mathrm{CV}(\%)$ & - & 13,13 & 11,29 & 14,11 & 6,40 & 5,06 \\
\hline DMS & - & 18,37 & 20,78 & 30,50 & 14,69 & 11,58 \\
\hline
\end{tabular}

${ }^{1 / \square}$ Acrescentou-se Energic a 0,5\% v/v.

2/ Acrescentou-se Assist a $0,05 \% \mathrm{v} / \mathrm{v}$.

3/ Acrescentou-se Dash a $0,5 \%$ v/v.

4/ Acrescentou-se Dytrol a $0,25 \%$ v/v.

* As médias seguidas pelas mesmas letras na coluna não diferem entre si pelo teste de Tukey a 5\% de probabilidade.

${ }^{1}$ Isopropilamina (Roundup original ${ }^{\circledR}$ ); ${ }^{2}$ Isopropilamina (Roundup Transorb ${ }^{\circledR}$ ); ${ }^{3}$ Amônia (Roundup WG $\left.{ }^{\circledR}\right) ;{ }^{4}$ Potássio (Zapp QI ${ }^{\circledR}$ ). "Formulações dos sais de glyphosate baseadas em $1080 \mathrm{~g}$ de equivalente ácido $\mathrm{ha}^{-1}$ (valores observados após o preparo da calda). 
Velho et al. (2004) também observaram excelente controle de $A$. tenella com [glyphosate + imazetapyr], sendo considerado uma ótima opção para operação de manejo da cultura da soja. Segundo Procópio et al. (2006), A. tenella foi controlada por glyphosate (1620 $\mathrm{g} \mathrm{ha}^{-1}$ ), contudo, o melhor controle foi obtido quando se adicionou imazethapyr $\left(100 \mathrm{~g} \mathrm{ha}^{-1}\right)$ ou chlorimuron-ethyl (10 ou $20 \mathrm{~g} \mathrm{ha}^{-1}$ ).

Diversos autores também relatam o controle de A. tenella por meio de aplicações de diferentes formulações de glifosato. Carvalho et al. (2004) observaram controle de A. tenella com aplicações de MON78239 $\left(1000, \quad 1500, \quad 3000\right.$ e $\left.4500 \quad \mathrm{~g}^{h^{-1}}\right)$ e MON78634 (500, 1000, 2000 e $3500 \mathrm{~g} \mathrm{ha}^{-1}$ ) na dessecação em pré-semeadura do milho.

Controle satisfatório de A. tenella com o herbicida MON78634 na dose $1500 \mathrm{~g}$ ha $^{-1}$ aplicado no estádio V5 do milho, foi observado por Ferreira Neto et al. (2004a). Excelente controle para A. tenella, acima de $96,00 \%$, foi observado por Kawaguchi et al. (2004) com aplicação do herbicida MON77280 em pós-emergência do algodão. Em acordo com esse resultado, também foi observado por Nishikawa et al. (2004) controle de $A$. tenella com aplicação de MON77280 em pós-emergência em soja RR e por Ferreira Neto et al. (2004b) em pósemergência de milho.

\section{Conclusões}

Todos os herbicidas aplicados em préemergência foram eficazes para o controle de Alternanthera tenella, proporcionando controle acima de $98 \%$ aos 28 DAA.

Os herbicidas fomesafen, lactofen e bentazon, aplicados em pós-emergência, não proporcionaram níveis aceitáveis de controle para A. tenella, em nenhuma das avaliações.
Os demais herbicidas pós-emergentes avaliados foram altamente eficazes, resultando num controle acima de $97 \%$ aos 28 DAA.

\section{Agradecimentos}

Aos funcionários do Núcleo de Estudos Avançados em Ciência das Plantas Daninhas (NAPD/UEM) Luis Machado Homem e Milton Luis da Silva, pelo auxílio na montagem e condução dos experimentos.

\section{Referências}

BERTIN, E.G.; ANDRIOLI, I.; CENTURION, J.F. Plantas de cobertura em pré-safra ao milho em plantio direto. Acta Scientiarum Agronomy, v.27, n.3, p.379386, 2005.

BEZUTTE, A.J.; CALEGARE, F.; ALVES, P.L.C.A.; PITELLI, R. Eficiência do herbicida oxyfluorfen, quando veiculado ao papel, no controle de algumas espécies daninhas. Planta Daninha, v.13, n.1, p.3945, 1995.

BRAZ, B.A.; NICOLAI, M.; SOARES, D.J. Avaliação da eficácia do trifloxysulfuron sodium + ametrina no controle de plantas daninhas e intoxicação às plantas de cana-deaçúcar de ano e meio (Saccharum spp.) In: CONGRESSO BRASILEIRO DA CIÊNCIA DAS PLANTAS DANINHAS, 23. Gramado, 2002. Resumos... Sociedade Brasileira da Ciência das Plantas Daninhas, 2002. p.498.

CARVALHO, J.E.B.; LOPES, L.C.; ARAÚJO, A.M.A. Ocorrência de plantas infestantes em três pomares de citros no estado de Sergipe. Magistra, v.17, n.3, p.148-153, 2005.

CARVALHO, J.A.; ARTINS, M.R.; MORAIS, T.R.; SANTOS, V.L.M; 
KAWAGUCHI, I.T.; BRITO, C.H. Eficácia e seletividade das formulações de glifosato MON 78239 e MON 78634 aplicados para dessecação na pré-semeadura do milho. In: CONGRESSO BRASILEIRO DA CIÊNCIA DAS PLANTAS DANINHAS, 10. São Pedro, 2002. Anais... Sociedade Brasileira da Ciência das Plantas Daninhas, 2004. 2p. CDROM.

CHRISTOFFOLETI, P.J.; MACHADO, F.C.; OLIVEIRA, A.M.F.; MONQUERO, P.A. Manejo de plantas daninhas na cultura de milho com os herbicidas sulfentrazone e carfentrazone-ethyl. R. Bras. Herb., v.3, n.2/3, p.145-153, 2002.

CORREIA, N.M.; SOUZA, I.F.; KLINK, U.P. Palha de sorgo associada ao herbicida imazamox no controle de plantas daninhas na cultura da soja em sucessão. Planta Daninha, v.23, n.3, p.483-489, 2005.

DUARTE, A.P.; DEUBER, R. Levantamento de plantas infestantes em lavouras de milho "safrinha" no estado de São Paulo. Planta Daninha, v.17, n.2, p.297-307, 1999.

FERREIRA, E.A.; PROCÓPIO, S.O.; SILVA, E.A.M.; SILVA，A.A.; RUFINO, R.J.N. Estudos anatômicos de folhas de espécies de plantas daninhas de grande ocorrência no Brasil. IV - Amaranthus deflexus, Amaranthus spinosus, Alternanthera tenella e Euphorbia hererophylla. Planta Daninha, v.21, n.2, p.263-271, 2003.

FERREIRA NETO, A.; FERREIRA, F.; KAWAGUCHI, I.T.; NISHIKAWA, M.N.; SCHON, M.A. Avaliação da eficácia e seletividade de MON78634 em comparação com outros herbicidas no controle de plantas daninhas em milho geneticamente modificado Roundup Ready (Evento NK603). In: CONGRESSO BRASILEIRO DA CIÊNCIA
DAS PLANTAS DANINHAS, 10. São Pedro, 2002. Anais... Sociedade Brasileira da Ciência das Plantas Daninhas, 2004a. 2p. CDROM.

FERREIRA NETO, A.; FERREIRA, F.; KAWAGUCHI, I.T.; NISHIKAWA, M.N.; SCHON, M.A. Avaliação da eficácia de MON14445 em diferentes doses e momentos de aplicação no controle de plantas daninhas na cultura do milho geneticamente modificado Roundup Ready (Evento NK603). In: CONGRESSO BRASILEIRO DA CIÊNCIA DAS PLANTAS DANINHAS, 10. São Pedro, 2002. Anais... Sociedade Brasileira da Ciência das Plantas Daninhas, 2004b. 2p. CD-ROM.

FOLONI, L.L.; BACHIEGA, A.L. Callisto (mesotrione) isolado ou associado a atrazine em pós-emergência no milho. In: CONGRESSO BRASILEIRO DA CIÊNCIA DAS PLANTAS DANINHAS, 23. Gramado, 2002. Resumos... Sociedade Brasileira da Ciência das Plantas Daninhas, 2002. p.656.

FREITAS, R.S.; FERREIRA, L.R.; BERGER, P.G.; SILVA, A.C.; CEDON, P.R.; SILVA, M.P. Manejo de plantas daninhas na cultura do algodoeiro com smetolachlor e trifloxysulfuron-sodium em sistema de plantio direto. Planta Daninha, v.24, n.2, p.311-318, 2006.

JAREMTCHUK，C.C.; CONSTANTIN，J.; OLIVEIRA JR.; R.S.; BIFFE, D.F.; ALONSO, D.G.; KAJIHARA, L.H.; TOLEDO, R.E.; FORLIVIO, D.M. Efeito residual de flumioxazin sobre a emergência de plantas daninhas em dois solos de textura distinta. In: CONGRESSO BRASILEIRO DA CIÊNCIA DAS PLANTAS DANINHAS, 25. Brasília, 2006. Anais... Sociedade Brasileira da Ciência das Plantas Daninhas, 2006. 3p. CD-ROM. 
KAWAGUCHI, I.T.; MONTEZUMA, M.C.; CAMPOSILVA, D.; PEREIRA, A.M.; FERREIRA NETO, A. Eficácia e seletividade do herbicida glifosato em aplicação isolada e seqüencial, sobre a cultura do algodão Roundup Ready (Evento 1445). In: IV CONGRESSO BRASILEIRO DE ALGODÃO, 2003. Goiânia, 2003. Resumos... $4 p$.

KAWAGUCHI, I.T.; FERREIRA NETO, A.; NISHIKAWA, M.N.; SCHON, M.A.; FERREIRA, F. Avaliação da eficácia do herbicida MON 77280 no controle de plantas daninhas na cultura do algodão geneticamente modificado Roundup Ready. In: CONGRESSO BRASILEIRO DA CIÊNCIA DAS PLANTAS DANINHAS, 10. São Pedro, 2002. Anais... Sociedade Brasileira da Ciência das Plantas Daninhas, 2004. 2p. CDROM.

KISSMANN, K.G.; GROTH, D. Plantas infestantes e nocivas. Tomo I 2 ed., São Paulo: Basf, 1999, 978p.

LACERDA, A.L.S. Fluxos de emergência e banco de sementes de plantas daninhas em sistemas de semeadura direta e convencional e curvas dose-resposta ao glyphosate. Piracicaba-SP, 2003. 141p. Tese (Doutorado em Fitotecnia) Escola Superior de Agricultura "Luiz de Queiroz".

LOPES, S.A.; MARCUSSI, S.; TORRES, S.C.Z.; SOUZA, V.; FAGAN, S.C.; FRANÇA, S.C. Weeds as alternative hosts of the citrus, coffee, and plum strains of Xylella fastidiosa in Brazil. Plant Disease, v.87, n.5, p.544-549, 2003.

LORENZI, H. Manual de identificação e controle de plantas daninhas: plantio direto e convencional 6 ed. Nova Odessa, SP: Instituto Plantarum, 2006, 337p.

NASCENTE, A.S.; PEREIRA, W.; MEDEIROS, M.A. Interferência de plantas daninhas na cultura do tomate para processamento. Hort. Bras., v.22, n.3, 2004.

NEGRISOLI, E.; COSTA, E.A.D.; COSTA, A.G.F.; VELINI, E.D. Interação de herbicidas dessecantes e de pré-emergência associados a adjuvante em plantio direto. Ecossistema, v.28, n.1/2, 2003.

NISHIKAWA, M.A.M.; FERREIRA NETO, A.; KAWAGUCHI, I.T.; SCHON, M.A. Avaliação da eficácia agronômica de MON77280 e herbicidas pré-emergentes no controle de plantas daninhas de soja geneticamente modificada Roundup Ready (Evento GTS 40-3-2). In: CONGRESSO BRASILEIRO DA CIÊNCIA DAS PLANTAS DANINHAS, 10. São Pedro, 2002. Anais... Sociedade Brasileira da Ciência das Plantas Daninhas, 2004. 2p. CDROM.

PEIXOTO, M.F.; PAULA de, J.M.; SILVA, O.A.B.; VIEIRA, S.S. Controle de plantas daninhas em pós-emergência na cultura do algodão. In: CONGRESSO BRASILEIRO DA CIÊNCIA DAS PLANTAS DANINHAS, 25. Brasília, 2006. Anais... Sociedade Brasileira da Ciência das Plantas Daninhas, 2006. 4p. CD-ROM.

PEREIRA, F.A.R.; VELINI, E.D. Sistemas de cultivo no cerrado e dinâmica de populações de plantas daninhas. Planta Daninha, v.21, n.3, p.355-363, 2003.

PROCÓPIO, S.O.; MENEZES, C.C.E.; PIRES, F.R.; BARROSO, A.L.L.; CARGNELUTTI FILHO, A.; RUDOVALHO, M.C.; MORAES, R.V.; 
SILVA, M.V.V.; CAETANO, J.O. Eficácia de imazethapyr e chlorimuron-ethyl em aplicações de pré-semeadura da cultura da soja. Planta Daninha, v.24, n.3, p.467-473, 2006.

RODRIGUES, B.N.; ALMEIDA, F.S. Guia de Herbicidas. Londrina: ed dos autores. 2005, 592p.

ROZANSKI, A.; COSTA, E.A.D.; CUNHA, A.V.V.; MALUF, E. Avaliação do herbicida bentazon + paraquat e associação com imazetaphyr e lactofen no controle de plantas daninhas na cultura de amendoim. In: CONGRESSO BRASILEIRO DA CIÊNCIA DAS PLANTAS DANINHAS, 10. São Pedro, 2002. Anais... Sociedade Brasileira da Ciência das Plantas Daninhas, 2004. 2p. CDROM.

SHIRATSUCHI, L.S. Mapeamento da variabilidade espacial das plantas daninhas com a utilização de ferramentas da agricultura de precisão. Piracicaba-SP, 2001. 96p. Tese (Mestrado em Fitotecnia), Escola Superior de Agricultura "Luiz de Queiroz".

TIMOSSI, P.C.; DURIGAN, J.C.; LEITE, C.J. Eficácia do glyphosate em plantas de cobertura. Planta Daninha, v.24, n.3, p.475480, 2006.

VALÉRIO, M.A.; FORNAROLLI, D.A.; MORAES, V.J.; CAETANO, E. Eficiência de herbicidas pós-emergentes aplicados isolados e em misturas no controle de plantas daninhas em diferentes estádios de crescimento. In: CONGRESSO BRASILEIRO DA CIÊNCIA DAS PLANTAS DANINHAS, 23. Gramado, 2002. Resumos... Sociedade Brasileira da Ciência das Plantas Daninhas, 2002. p.437.
VELHO, G.F.; IKEDA, M.; FELIPPE, J.M. Avaliação do herbicida BAS $686 \mathrm{H}$ aplicado na dessecação de plantas daninhas em préplantio da cultura da soja (Glycine max). In: CONGRESSO BRASILEIRO DA CIÊNCIA DAS PLANTAS DANINHAS, 10. São Pedro, 2002. Anais... Sociedade Brasileira da Ciência das Plantas Daninhas, 2004. 2p. CDROM. 